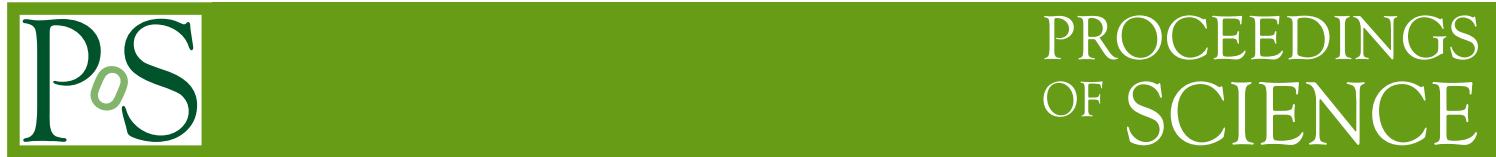

\title{
FLAG phase 2: status and prospects
}

\author{
Gilberto Colangelo*i \\ Albert Einstein Center for fundamental physics \\ Institute for theoretical physics \\ University of Bern \\ Sidlerstr. 5, 3012 Bern \\ E-mail: gilberto@itp.unibe.ch
}

In this talk I presented the FLAG initiative, discussed the history of the project, its aim and scope. After completing the first review in 2010, we decided to extend the review to more quantities and to involve a larger group of people. I have illustrated the phase 2 of the project, its new structure and the schedule for the release of the next review.

The 30th International Symposium on Lattice Field Theory

June 24-29, 2012

Cairns, Australia

\footnotetext{
* Speaker.

†n behalf of FLAG - Flavour Lattice Averaging Group.
}

Work supported in part by the Swiss National Science Foundation. 


\section{Introduction}

As much as it is fascinating per se to understand and calculate observables in a system which is strongly interacting, one of the most important goals of the lattice method is the calculation of hadronic matrix elements. There are many examples of quantities for which there is no alternative to lattice QCD if one wants a reliable error estimate and the possibility to systematically improve the calculation. And when there are alternative methods, it is anyway a very good check to be able to compare to lattice calculations. Many hadronic matrix elements represent a sort of "background" in the search for possible deviations from the Standard Model (SM) prediction and it is essential to have a precise calculation and reliable error estimate in order to fully exploit experimental measurements. The quantity $\varepsilon^{\prime} / \varepsilon$ is perhaps the best known example of a very difficult experimental measurement, which took more than twenty years of efforts before getting to the desired goal, and which until now could not been used as a test of the SM, because a precise enough calculation of the relevant hadronic matrix elements is still lacking. The recent measurement of $\Delta A_{C P}$ by $\mathrm{LHCb}$ [1] provides another striking example, but there are many more, with even more to come, especially in $B$ and $D$ physics.

In most of the cases of interest the lattice calculations are still quite challenging and the desired results cannot yet be found in textbooks. If an experimentalist or a theorist wants to have an input from lattice QCD he or she need to find it in research papers and or talk to lattice colleagues. Reading the lattice papers and properly extracting the desired information is certainly a challenge for a nonexpert. Given the importance of what is at stake (the discovery of physics beyond the SM) an effort should be made to make sure that the results of lattice calculations are used correctly by the "end users". This effort is a responsibility of the lattice community.

One could of course argue that this duty belongs to each individual lattice group and should be fulfilled in each paper which is written - but it is a fact that most of the time the same quantity is calculated by different groups with different methods which address differently the various systematic effects, so that a comparison of various calculations can provide additional information which cannot be found in any of the individual papers. The effort to summarize the status of the lattice calculations of the various quantities of interest is worthwhile per se. I believe that the view presented here is shared by most of the lattice community already since long. The problem is that this is a nontrivial enterprise, especially if one wants to produce something which is and is perceived as fair and representative of the actual status of the field.

In the following pages I will describe the FLAG initiative and to a lesser extent (especially for what concerns the history, for obvious reasons) the similar initiative of Laiho, Lunghi and Van de Water. Both initiatives led to reviews and webpages which have been read and used by many people. The feedback received has been overall quite positive and has pushed us to continue this activity, extend it and improve it. Most importantly, the two groups have now merged. In the following I will refer to the first (second) FLAG review as FLAG-1(2) and to the collaboration as FLAG, when necessary specifying whether phase 1 or 2.

\section{FLAG phase 1}

The FLAG initiative started in the framework of the European network FLAVIAnet which 
existed between 2006 and 2010 - indeed the acronym stays for FLAVIAnet Lattice Averaging Group. The focus of the network was on flavour physics and the aim was to bring new progress in the field by combining the efforts of experimentalists and theorists and, among the theorists, between those using effective field theories and lattice. At the beginning of the network's period it was decided to form a working group whose aim was to make a review of lattice results. In order to have a chance to reach this goal in a finite time we decided to restrict the quantities under consideration to a rather small subset: those concerning the three light quarks, at zero temperature and zero baryon number. More precisely, we concentrated on:

1. light quark masses;

2. $f_{+}(0)$ and $f_{K} / f_{\pi}$ and the determination of $V_{u s}$ and $V_{u d}$;

3. the low energy constants of the strong chiral Lagrangian;

4. $B_{K}$.

The working group consisted of twelve people, namely (the affiliation and lattice group refer to phase 1 - in some cases they have changed in the meanwhile, see section 5):

Stephan Dürr (Jülich, BMW)

Andreas Jüttner (Southampton, RBC/UKQCD)

Laurent Lellouch (Marseille, BMW)

Heiri Leutwyler (Bern)

Vittorio Lubicz (Rome 3, ETM)

Silvia Necco (CERN, Alpha)

Chris Sachrajda (Southampton, RBC/UKQCD)

Silvano Simula (Rome 3, ETM)

Tassos Vladikas (Rome 2, Alpha and ETM)

Urs Wenger (Bern, ETM)

Hartmut Wittig (Mainz, Alpha)

and myself. For obvious reasons all the members were European and were working in European lattice collaborations with the exception of two people: Heiri Leutwyler and myself, who did not (and still don't) belong to any lattice collaborations.

After having formed the group at the end of 2007 we started work with a meeting in Bern in March 2008. The progress was slower than originally foreseen (which is not surprising) and the review was completed and made public on the arXiv at the end of 2010. It contained results which had been published until June 30 2010. After being accepted for publication in the European Physical Journal C in early 2011 we decided to update it to results published until February 28 2011, and this is the version which appeared in EPJC [2]. The results are also available on the web at the address: itpwiki.unibe.ch/flag

\subsection{Quality criteria}

At the beginning of the work of FLAG it was decided to establish a number of quality criteria which would then be used as guidelines when preparing the summaries for the individual quantities. 
These quality criteria had to address the main systematic effects affecting a lattice calculations, namely:

- unphysical quark masses;

- finite volume;

- finite lattice spacing.

Moreover for some quantities, what is calculated on the lattice needs to be multiplied by a renormalization factor, before being able to compare this with what is extracted from experiment. The calculation of the renormalization factors can be done either nonperturbatively on the lattice, or in perturbation theory - this choice also affects the reliability of the result and therefore must be included in the list of sources of systematic effects.

Each calculation has been scrutinized in order to establish how well each of these systematic effects had been addressed. The conclusion has been expressed in a simplified form by assigning each calculation (for each systematic effect) to one of the following broad classes:

1. the systematic effect has been addressed, estimated reliably and convincingly shown to be under control;

2. a reasonable attempt has been made which could be improved;

3. no or a clearly unsatisfactory attempt to estimate the systematic error has been made.

To make this classification easily readable it has been color coded ( $\star$ for case 1 ., $\bullet$ for case 2 . and - for case 3.) and presented in a table together with the numerical outcome of the calculation. An example of such a table will be shown in the next section.

We decided very early on to base our classification on a priori criteria, and translated our broad definitions above into explicit conditions as follows ${ }^{1}$

- Chiral extrapolation:

$\star M_{\pi, \min }<250 \mathrm{MeV}$

- $250 \mathrm{MeV} \leq M_{\pi, \min } \leq 400 \mathrm{MeV}$

- $M_{\pi, \min }>400 \mathrm{MeV}$

It is assumed that the chiral extrapolation is done with at least a three-point analysis - otherwise this will be explicitly mentioned in a footnote. In case of nondegeneracies among the different pion states $M_{\pi, \min }$ stands for a root-mean-squared (RMS) pion mass.

- Continuum extrapolation:

$\star 3$ or more lattice spacings, at least 2 points below $0.1 \mathrm{fm}$

- 2 or more lattice spacings, at least 1 point below $0.1 \mathrm{fm}$

- otherwise

It is assumed that the action is $O(a)$-improved, i.e. the discretization errors vanish quadratically with the lattice spacing - otherwise this will be explicitly mentioned in a footnote.

\footnotetext{
${ }^{1}$ In order to avoid any possible confusion these definitions - i.e. the complete bulleted list - are taken verbatim from Ref. [2].
} 
Moreover the colour coding criteria for non-improved actions change as follows: one lattice spacing more needed.

- Finite-volume effects:

$\star \quad M_{\pi, \min } L>4$ or at least 3 volumes

- $M_{\pi, \min } L>3$ and at least 2 volumes

- otherwise

These criteria apply to calculations in the $p$-regime, and it is assumed that $L_{\min } \geq 2 \mathrm{fm}$, otherwise this will be explicitly mentioned in a footnote and a red square will be assigned. In case of nondegeneracies among the different pion states $M_{\pi \text {,min }}$ stands for a root-meansquared (RMS) pion mass.

- Renormalization (where applicable):

$\star$ non-perturbative

- 2-loop perturbation theory

- otherwise

- Running (where applicable): For scale-dependent quantities, such as quark masses or $B_{K}$, it is essential that contact with continuum perturbation theory can be established. Various different methods are used for this purpose: Regularization-independent Momentum Subtraction (RI/MOM), Schrödinger functional, direct comparison with (resummed) perturbation theory. In the case of the quark masses, a further approach has been proposed recently: determination of $m_{s}$ via the ratio $m_{c} / m_{s}$. Quite irrespective of the particular method used, the uncertainty associated with the choice of intermediate renormalization scales in the construction of physical observables must be brought under control. This is best achieved by performing comparisons between non-perturbative and perturbative running over a reasonably large range of scales. These comparisons were initially only made in the Schrödinger functional (SF) approach, but are now also being performed in RI/MOM schemes.

In the framework of the Schrödinger functional, the comparison of the lattice results for the relevant renormalization factors with perturbation theory has thoroughly been explored. Among the calculations relying on the RI/MOM framework, the most recent ones are aiming for a level of control over running and matching which is of comparable quality. However, since these approaches are new, we postpone the formulation of quantitative criteria until the systematics associated with their use is better understood. We mark those data for which information about non-perturbative running checks is available and give some details, but do not attempt to translate this into a colour-code.

This classification already provides a good overview of the status of lattice calculations for a certain quantity, but these yet need to be combined into the "current lattice number" for that quantity. If there is more than one lattice calculations of the same quantity, which can be considered reliable, it makes sense to average these, or to provide an estimate based on these. Once more, it was decided to have a priori criteria for what to propose as the "current lattice number".

First of all, any calculation which had a red tag was excluded from any averaging procedure. We also decided to consider only calculations which had been peer-reviewed - excluding in this 
way any conference (and in particular the yearly Lattice Conference) proceedings, unless these presented an obvious and uncontroversial update of a calculation already published in a peer-reviewed article. Finally, it was decided to exclude any quenched calculations and to summarize and average separately lattice calculations with $N_{f}=2$ and $N_{f}=2+1$.

Whenever a set of different numbers affected by both statistical and systematic errors must be averaged - irrespective of whether these come from theoretical calculations or experimental measurements - one is faced with the problem of how to deal with the systematic error. A single correct recipe does not exist, but there are several working ones (e.g. the one adopted by the PDG [3]). In FLAG phase 1 we agreed to adopt the PDG one, which is to sum in quadrature the statistical and systematic error and proceed with calculating the average in the standard way, checking a posteriori whether this is sensible. In particular we checked whether $\chi_{\min }^{2} / \mathrm{dof} \leq 1$ and if

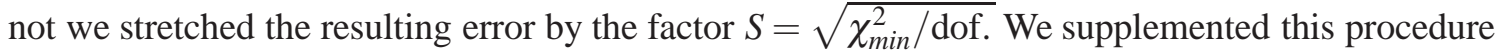
with the check whether the final error of the average was smaller than the smallest systematic error of any calculation entering the average. If this is the case we take the smallest systematic error as our final estimate of the error.

These quality criteria did not exempt us from applying some individual judgment to the analysis of the various calculations, and indeed in some cases we found it necessary to make exceptions, which have been discussed and explained in the paper. In particular, in some cases we did not find that the plain average of the lattice calculations which had satisfied our quality criteria provided a conservative enough uncertainty. In such cases instead of calculating an average we provided an estimate.

\subsection{Beyond averages}

The first FLAG review did not offer only averages. Whenever we identified issues which needed to be better discussed with respect to what was available in the literature, we tried to provide the corresponding discussion. For example, lattice calculation have begun only recently to consider seriously electromagnetric contributions to hadron masses, and a thorough discussion of what are the relevant parameters to be determined and what was known about these, both from lattice calculations or the phenomenology was lacking. This has been provided in [2]. Also, the determinations of $f_{+}(0)$ and $f_{K} / f_{\pi}$ lead to a determination of $\left|V_{u s}\right|$ and $V_{u s} / V_{u d}$, which in turn allow a test of the unitarity of the first row of the CKM matrix. On the other hand, once no signs of violations of unitarity emerge, one can turn the problem around and assume unitarity to test the consistency of the lattice calculations of $f_{+}(0)$ and $f_{K} / f_{\pi}$. Since these involve very different systematic effects and the assumption of unitarity reduces substantially the uncertainty in the determination of $\left|V_{u s}\right|$ and $\left|V_{u d}\right|$, this provides a very stringent consistency test of these lattice calculations. This observation is new and has also been provided in the FLAG review.

Finally, we have provided either completely new or more conveniently written two-loop formulae in $\chi \mathrm{PT}$ and a glossary of lattice terms and a summary of the main features of the most commonly used actions.

\section{The FLAG-1 review of lattice-based $V_{u s}$ and $V_{u d}$ determination}

In order to show what the outcome of the procedure just described looks like, I will briefly 


\begin{tabular}{|c|c|c|c|c|c|c|c|}
\hline Collaboration & Ref. & $N_{f}$ & Ẽ & co & $0^{\circ}$ & si & $f_{+}(0)$ \\
\hline RBC/UKQCD 10 & [4] & $2+1$ & A & - & $\mathbf{\square}$ & $\star$ & $0.9599(34)\left({ }_{-47}^{+31}\right)(14)$ \\
\hline RBC/UKQCD 07 & [5] & $2+1$ & A & $\bullet$ & च & $\star$ & $0.9644(33)(34)(14)$ \\
\hline ETM 10D & [6] & 2 & $\mathrm{C}$ & - & $\star$ & - & $0.9544(68)_{\text {stat }}$ \\
\hline ETM 09A & [7] & 2 & A & - & - & - & $0.9560(57)(62)$ \\
\hline QCDSF 07 & [8] & 2 & C & [ & [ & $\star$ & $0.9647(15)_{\text {stat }}$ \\
\hline RBC 06 & [9] & 2 & A & [ & [ & $\star$ & $0.968(9)(6)$ \\
\hline JLQCD 05 & [10] & 2 & $\mathrm{C}$ & घ & ש & $\star$ & $0.967(6), 0.952(6)$ \\
\hline
\end{tabular}

Table 1: Colour code for the data on $f_{+}(0)$.

describe the content of the review for what concerns the determination of $V_{u s}$ and $V_{u d}$. Tables and figures presented here exactly coincide with those in [2].

Determining $V_{u s}$ and $V_{u d}$ requires lattice input for two hadronic observables, the flavourchanging $K \pi$ vector form factor at zero momentum transfer, usually denoted by $f_{+}(0)$ and the ratio of the decay constants of the kaon and of the pion, $f_{K} / f_{\pi}$. Experimental measurements allow a precise extraction of the product $\left|V_{u s}\right| f_{+}(0)$ and the ratio $\left|V_{u s} f_{K}\right| /\left|V_{u d} f_{\pi}\right|$ [27]:

$$
\left|V_{u s}\right| f_{+}(0)=0.2163(5), \quad\left|\frac{V_{u s} f_{K}}{V_{u d} f_{\pi}}\right|=0.2758(5),
$$

so that with the mentioned lattice input one can obtain $\left|V_{u s}\right|$ and the ratio $\left|V_{u s} / V_{u d}\right|$. The status of lattice calculations for $f_{+}(0)$ is summarized in table 1 and the one for $f_{K} / f_{\pi}$ in table 2. The situation is also summarized in figure 1 where, as far as $f_{+}(0)$ is concerned, also phenomenological estimates based on $\chi \mathrm{PT}$ are shown. The figures show that the lattice determinations of the two quantities are broadly consistent among each other, and that the overall consistency even improves if one considers only calculation which do not have red tags (in the figures these are shown in green - which means that the calculation may have both green and yellow tags, but not red ones). Notice also that for $f_{+}(0)$ there is a single $N_{f}=2+1$ determination (the two data points are from the same collaboration, and the second one supersedes the first) and that this does have a red tag as it does not fulfill our a priori quality criteria for what concerns discretization effects. Nonetheless, in view of the fact that this source of systematic effect has been estimated to be subdominant in [5] and that the second paper [4] confirmed this estimate after adding a second lattice spacing, it was decided to take the latter as the FLAG number for the $N_{f}=2+1$ determination of $f_{+}(0)$ (this is one of the few exceptions to the FLAG rules which can be found in the review). As this $N_{f}=2+1$ determination agrees quite well with the $N_{f}=2$ which is free from red tags, the final FLAG estimate for $f_{+}(0)$ is

$$
f_{+}(0)=0.956(8) \text {, }
$$




\begin{tabular}{|c|c|c|c|c|c|c|c|}
\hline Collaboration & Ref. & $N_{f}$ & 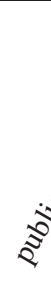 & ह & ثئ & s & $f_{K} / f_{\pi}$ \\
\hline ETM 10E & {$[11]$} & $2+1+1$ & $\mathrm{C}$ & - & - & - & $1.224(13)_{\text {stat }}$ \\
\hline MILC 10 & [12] & $2+1$ & $\mathrm{C}$ & ○ & $\star$ & $\star$ & $1.197(2)\left(\begin{array}{l}+3 \\
-7\end{array}\right)$ \\
\hline RBC/UKQCD 10A & [13] & $2+1$ & $\mathrm{P}$ & $\bullet$ & - & $\star$ & $1.204(7)(25)$ \\
\hline BMW 10 & [14] & $2+1$ & A & $\star$ & $\star$ & $\star$ & $1.192(7)(6)$ \\
\hline JLQCD/TWQCD 09A & [15] & $2+1$ & $\mathrm{C}$ & $\bullet$ & $\mathbf{\square}$ & $\mathbf{\square}$ & $1.210(12)_{\text {stat }}$ \\
\hline MILC 09A & [16] & $2+1$ & $\mathrm{C}$ & $\bullet$ & $\star$ & $\star$ & $1.198(2)\left({ }_{-8}^{+6}\right)$ \\
\hline MILC 09 & [17] & $2+1$ & A & - & $\star$ & $\star$ & $1.197(3)\left({ }_{-13}^{+6}\right)$ \\
\hline Aubin 08 & {$[18]$} & $2+1$ & $\mathrm{C}$ & - & - & ○ & $1.191(16)(17)$ \\
\hline PACS-CS 08, 08A & {$[19,20]$} & $2+1$ & A & $\star$ & $\mathbf{\square}$ & $\boldsymbol{\square}$ & $1.189(20)$ \\
\hline RBC/UKQCD 08 & [21] & $2+1$ & A & - & 口 & $\star$ & $1.205(18)(62)$ \\
\hline HPQCD/UKQCD 07 & {$[22]$} & $2+1$ & A & ○ & $\star$ & - & $1.189(2)(7)$ \\
\hline NPLQCD 06 & {$[23]$} & $2+1$ & A & ○ & $\square$ & $\mathbf{\square}$ & $1.218(2)\left({ }_{-24}^{+11}\right)$ \\
\hline MILC 04 & {$[24]$} & $2+1$ & A & $\star$ & - & - & $1.210(4)(13)$ \\
\hline ETM 10D & [6] & 2 & $\mathrm{C}$ & ○ & $\star$ & $\bullet$ & $1.190(8)_{\text {stat }}$ \\
\hline ETM 09 & {$[25]$} & 2 & A & ○ & $\star$ & - & $1.210(6)(15)(9)$ \\
\hline QCDSF/UKQCD 07 & {$[26]$} & 2 & $\mathrm{C}$ & ○ & - & $\star$ & $1.21(3)$ \\
\hline
\end{tabular}

Table 2: Colour code for the data on $f_{K} / f_{\pi}$.

which covers both results.

For $f_{K} / f_{\pi}$ it was instead possible to make two independent averages, with more $N_{f}=2+1$ calculations available than $N_{f}=2$.

$$
\begin{array}{ll}
f_{K} / f_{\pi}=1.193(5), & \left(\text { direct }, N_{f}=2+1\right), \\
f_{K} / f_{\pi}=1.210(6)(17), & \left(\text { direct }, N_{f}=2\right) .
\end{array}
$$

The above results for $f_{+}(0)$ and $f_{K} / f_{\pi}$ can be converted into a corresponding range for the CKM matrix elements $V_{u d}$ and $V_{u s}$, using the experimental determinations. For $N_{f}=2+1$ calculations the range for $f_{+}(0)$ is mapped into the interval $V_{u s}=0.2255(14)$, depicted as a horizontal gray band in Figure 2, while the one for $f_{K} / f_{\pi}$ in (3.3) is converted into $V_{u s} / V_{u d}=0.2312(11)$, shown as a green band. The red curve is the intersection of these two bands and represents the $68 \%$ likelihood contour, obtained by treating the above two results as independent measurements. Values of $V_{u s}$, $V_{u d}$ in the region enclosed by this contour are consistent with the lattice data for $N_{f}=2+1$, within one standard deviation. In particular, the plot shows that the nuclear $\beta$ decay result for $V_{u d}$ is perfectly consistent with these data.

Repeating the exercise with the $N_{f}=2$ results leads to the dashed ellipse. The figure thus indicates that the data are consistent within errors and that the quenching of the strange quark does not lead to visible effects, at the present level of accuracy. 

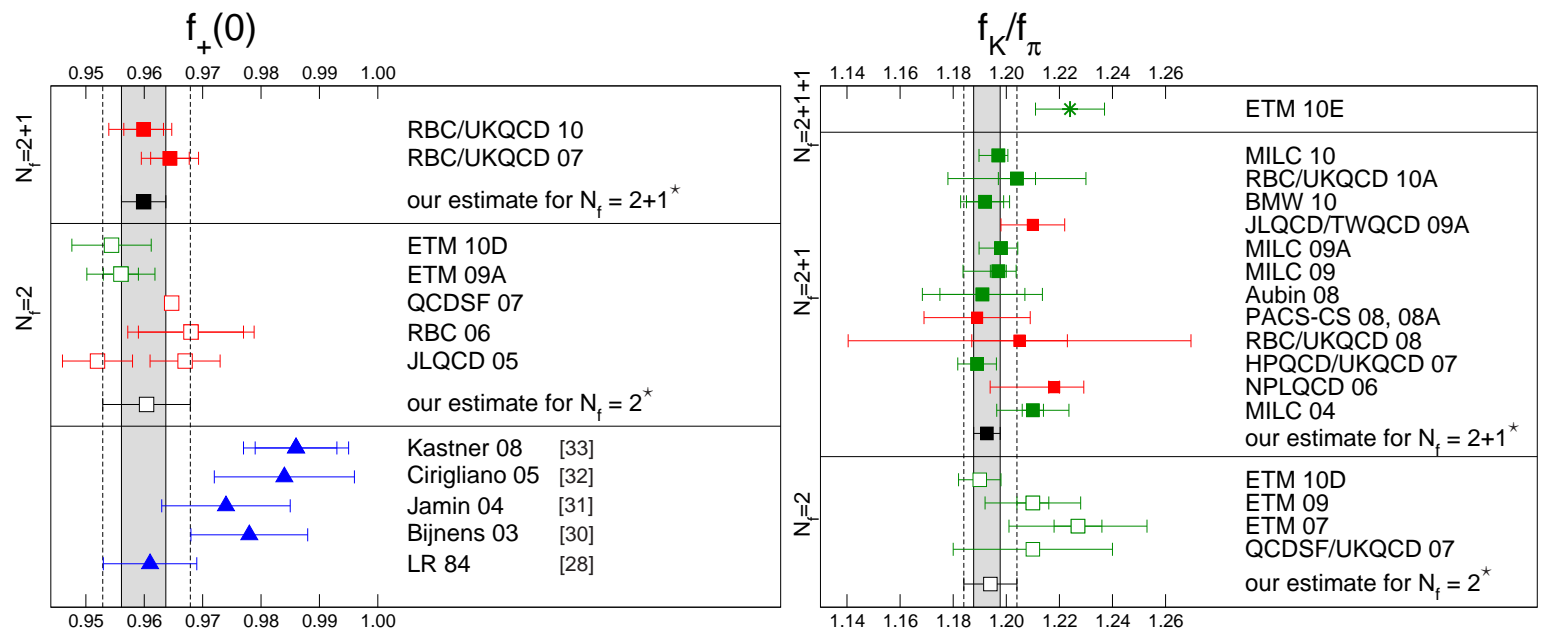

* Estimates obtained from an analysis of the lattice data within the Standard Model.

Figure 1: Comparison of lattice results (red squares) for $f_{+}(0)$ and $f_{K} / f_{\pi}$ with various model estimates based on $\chi \mathrm{PT}$ (blue triangles). Full and empty squares represent simulations with $N_{f}=2+1$ and $N_{f}=2$, respectively. The vertical bands indicate our estimates. *

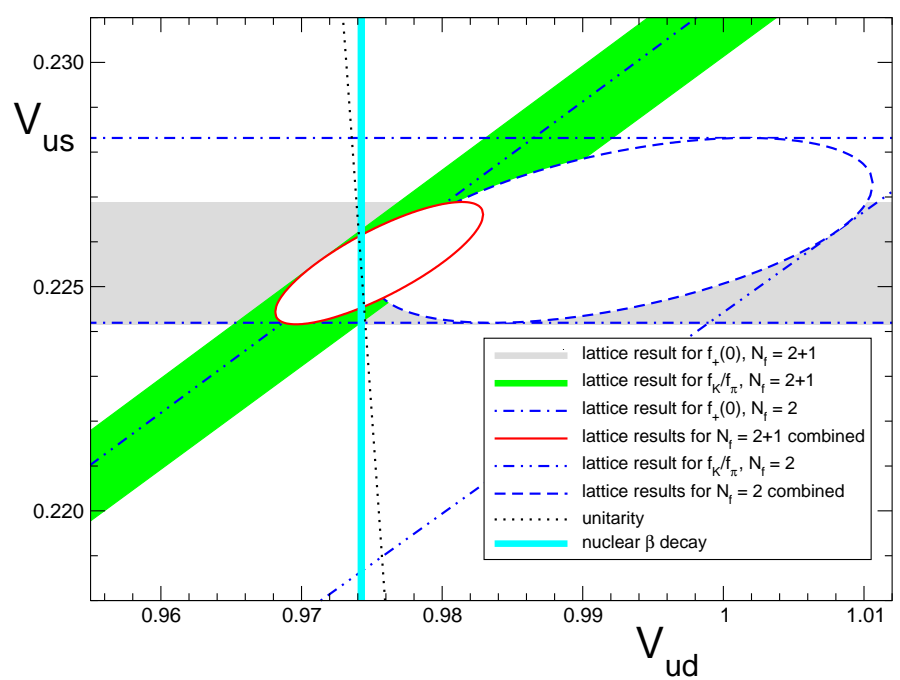

Figure 2: The plot compares the information for $\left|V_{u d}\right|,\left|V_{u s}\right|$ obtained on the lattice with the experimental result extracted from nuclear $\beta$ transitions. The dotted arc indicates the correlation between $\left|V_{u d}\right|$ and $\left|V_{u s}\right|$ that follows if the three-flavour CKM-matrix is unitary.

\subsection{Consistency test assuming unitarity}

The Standard Model implies that the CKM matrix is unitary. The precise experimental constraints quoted in (3.1) and the unitarity condition then reduce the four quantities $\left|V_{u d}\right|,\left|V_{u s}\right|, f_{+}(0)$, $f_{K} / f_{\pi}$ to a single unknown: any one of these determines the other three within narrow uncertainties.

In Figure 3 which is also taken from [2] we show that the results obtained for $\left|V_{u s}\right|$ and $\left|V_{u d}\right|$ from the data on $f_{K} / f_{\pi}$ (red squares) are quite consistent with the determinations via $f_{+}(0)$ (red triangles). We have even calculated the corresponding average values, after applying the restriction 


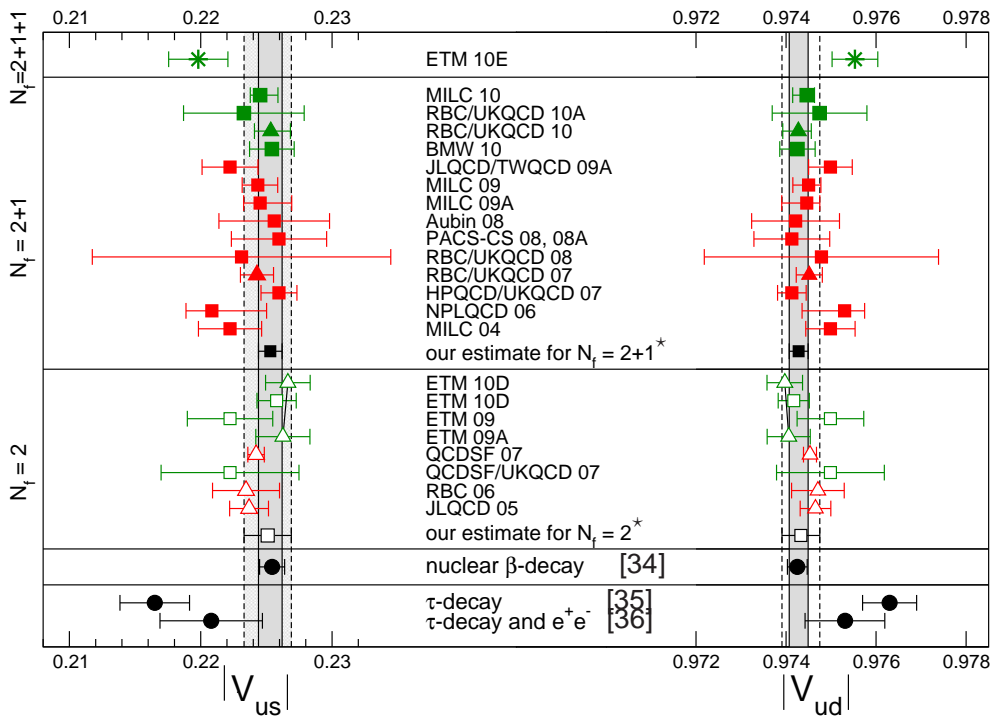

* Estimates obtained from an analysis of the lattice data within the Standard Model.

Figure 3: Results for $\left|V_{u s}\right|$ and $\left|V_{u d}\right|$ that follow from the lattice data for $f_{+}(0)$ (red triangles) and $f_{K} / f_{\pi}$ (red squares), on the basis of the assumption that the CKM matrix is unitary. The black squares and the bands represent our estimates, obtained by combining these two different ways of measuring $\left|V_{u s}\right|$ and $\left|V_{u d}\right|$ on a lattice. For comparison, the figure also indicates the results obtained if the data on nuclear $\beta$ decay and $\tau$ decay are analyzed within the Standard Model.

to the best determinations as discussed above.

The fact that the results from the four $N_{f}=2+1$ data sets RBC/UKQCD 10 [4], BMW 10 [14], MILC 10 [12] and HPQCD/UKQCD 07 [22] are consistent with each other provides a thorough reliability test of the lattice work. Treating the four sets as independent measurements, and applying the standard averaging procedure, we obtain a fit of good quality, with $\left|V_{u s}\right|=0.2253(6)$ and $\chi^{2}=$ 0.6 for 4 data points and 1 free parameter. The standard procedure underestimates the systematic uncertainties also in this case. Applying the prescription discussed above, we arrive at a somewhat larger error: $\left|V_{u s}\right|=0.2253(9)$. This result is indicated on the left hand side of Fig. 3 by the narrow vertical band. The broader band shows the corresponding value for $N_{f}=2$ (standard error analysis, $\left|V_{u s}\right|=0.2253(17)$, with $\chi^{2}=1.2$ for 2 data points and 1 free parameter, $S=1.09$ ). The figure shows that the result obtained for the data with $N_{f}=2$ is perfectly consistent with the one found for $N_{f}=2+1$.

One can also repeat the same exercise for $\left|V_{u d}\right|$ instead of $\left|V_{u s}\right|$. Again, the result $\left|V_{u d}\right|=$ $0.97428(21)$ which follows from the lattice data with $N_{f}=2+1$ is perfectly consistent with the value $\left|V_{u d}\right|=0.97433(42)$ obtained from those with $N_{f}=2$. The reduction of the uncertainties in the result for $\left|V_{u d}\right|$ due to CKM unitarity is to be expected from Figure 2: the unitarity condition reduces the region allowed by the lattice results to a nearly vertical interval.

The same exercise can be repeated solving for $f_{+}(0)$ and for $f_{K} / f_{\pi}$ and the result can be seen in Table 3. The results obtained by analyzing the lattice data after assuming unitarity are collected in the upper half of Table 3. In the lower half of this table one can find analogous results 


\begin{tabular}{llllll} 
& Ref. & \multicolumn{1}{c}{$\left|V_{u s}\right|$} & \multicolumn{1}{c}{$\left|V_{u d}\right|$} & $f_{+}(0)$ & $f_{K} / f_{\pi}$ \\
\hline \hline$N_{f}=2+1$ & & $0.2253(9)$ & $0.97428(21)$ & $0.9599(38)$ & $1.1927(50)$ \\
\hline$N_{f}=2$ & & $0.2251(18)$ & $0.97433(42)$ & $0.9604(75)$ & $1.194(10)$ \\
\hline \hline$\beta$-decay & {$[34]$} & $0.22544(95)$ & $0.97425(22)$ & $0.9595(46)$ & $1.1919(57)$ \\
$\tau$-decay & {$[35]$} & $0.2165(26)$ & $0.9763(6)$ & $0.999(12)$ & $1.244(16)$ \\
$\tau$-decay & {$[36]$} & $0.2208(39)$ & $0.9753(9)$ & $0.980(18)$ & $1.218(23)$ \\
\hline \hline
\end{tabular}

Table 3: The upper half of the table shows our final results for $\left|V_{u s}\right|,\left|V_{u d}\right|, f_{+}(0)$ and $f_{K} / f_{\pi}$, which are obtained by analyzing the lattice data within the Standard Model. For comparison, the lower half lists the values that follow if the lattice results are replaced by the experimental results on nuclear $\beta$ decay and $\tau$ decay, respectively.

found by working out the consequences of CKM-unitarity for the experimental values of $\left|V_{u d}\right|$ and $\left|V_{u s}\right|$ obtained from nuclear $\beta$-decay and $\tau$-decay, respectively. The comparison shows that the lattice result for $\left|V_{u d}\right|$ not only agrees very well with the totally independent determination based on nuclear $\beta$ transitions, but is also remarkably precise. The $\tau$-decay based determination shows instead a discrepancy with all the others. The disagreement is reduced considerably if the analysis of the $\tau$ data is supplemented with experimental results on electroproduction [36]: the discrepancy then amounts to little more than one standard deviation.

\section{The Laiho, Lunghi and Van de Water project}

A similar effort to FLAG was started in 2009 by Jack Laiho, Enrico Lunghi and Ruth Van de Water (LLVdW) and published the same year [37]. The main differences to the criteria adopted by FLAG were the following:

- only $N_{f}=2+1$ calculations were considered;

- all papers published on the arXiv or in journals were considered (including plain conference proceedings);

- systematic and statistical errors were summed in quadrature and averaged. However, whenever a single source of correlation between two calculations was identified it was conservatively assumed that the level of correlation was $100 \%$;

- effort more directly aimed at the determination of CKM matrix elements and both light and heavy quark quantities were considered from the start.

Despite the differences to the criteria adopted in FLAG, the conclusions, where a comparison was possible, were quite similar. LLVdW have published their results on a web page

(www. latticeaverages.org) which is also quite popular. 


\section{FLAG phase 2}

After completing the first review, the original FLAG group discussed the future of the initiative. It was very soon decided to extend the project in terms of quantities considered, people to be involved, both for what concerned their geographical location and the lattice collaborations they belonged to. Moreover, the experience made in managing the activities of a medium-sized group of authors of a review, taught us that it was necessary to have a more formal structure and a set of rules to which all members of the group had to abide, in order to make the inner workings of FLAG-2 function smoothly. After some preparatory work we invited an existing member (Chris Sachrajda) and two new members (Claude Bernard and Sinya Aoki) to be part of the advisory board of FLAG2 and they all immediately accepted. The task of the advisory board is to overview the activities of FLAG-2 and provide advice for what concerns important strategic decision. Among these, one of the most important ones is the choice of new members. During the 2011 Lattice conference we held meetings between FLAG members and the newly formed advisory board to discuss both the set of rules proposed and also a which new members would be invited. In particular it was decided to invite Laiho, Lunghi and Van de Water to join the phase 2 of FLAG and make out of the two groups into a larger one. The invitation was accepted.

The complete list of members of FLAG-2 and their assignment to Working Groups is:

- Advisory Board (AB):

S. Aoki, C. Bernard, C. Sachrajda

- Editorial Board (EB):

G. Colangelo, H. Leutwyler, T. Vladikas, U. Wenger

- Working Groups

- Quark masses

L. Lellouch, T. Blum, V. Lubicz

- $V_{u s}, V_{u d}$

- LEC

A. Jüttner, T. Kaneko, S. Simula

$-B_{K}$

S. Dürr, H. Fukaya, S. Necco

$-\alpha_{s}$

H. Wittig, J. Laiho, S. Sharpe

- $f_{B}, B_{B}$

R. Sommer, R. Horsley, T. Onogi

- $B \rightarrow H \ell v$

A. El Khadra, Y. Aoki, M. Della Morte

R. Van de Water, E. Lunghi, C. Pena, J. Shigemitsu

As it is seen from the list above, the only other entity inside FLAG-2 beyond the advisory board and the various working groups is the editorial board. This formalizes what happened already within FLAG phase 1: the work of putting together the various sections, moderating the discussions and in general coordinating all efforts had to a large extent been made by Heiri Leutwyler, Urs Wenger and myself. It proved essential that we were located in the same place in order to ease communication on a daily basis and to allow the organization of small meetings when necessary. Given the increase in size of the whole project we found it necessary to correspondingly enlarge the EB and have now been joined by Tassos Vladikas.

The most important general plans and internal rules of FLAG-2 are the following:

- the next review is due by the beginning of 2013 and will take into account all papers published until December 31 2012; 
- we plan to regularly update the webpage, yearly or even more often;

- a new published review will appear every second year.

- internal FLAG rules

- members of the $\mathrm{AB}$ have a 4-year mandate (to avoid a simultaneous change of all members at once, the current members will have a shorter mandate)

- the composition of the $\mathrm{AB}$ will respect a geographical balance among the main areas in which lattice collaborations are active: one member will come from America, one from Asia/Oceania and one from Europe ;

- the mandate of regular members is not limited in time, but we expect that a certain rotation will happen naturally;

- whenever a replacement becomes necessary this has to at least keep, and possibly improve the balance of FLAG;

- in all working groups the three members must belong to three different lattice collaborations ${ }^{2}$

- a paper is in general not reviewed (and color-coded) by an author;

- lattice collaborations not represented inside FLAG will be asked to check whether the color coding of their calculation is correct.

Work on the new review was started during a kick-off meeting held in Les Houches in Spring 2012, with participation of about two thirds of all FLAG members. A first draft of the new review is being circulated for internal discussion. This will still be worked on during the coming few months and will be published in early 2013 according to our plans.

\section{Conclusions}

The necessity to provide a review of lattice calculations of quantities of phenomenological interest has long been felt inside and outside the lattice community. Two initiatives have been started in recent years and have produced useful reviews and webpages [2, 37]. The two groups have now merged into a larger initiative whose current status, goals and internal rules I have briefly described here. The first review of the FLAG-2 initiative is due by early 2013. We believe that this nontrivial enterprise is important and very beneficial (if carried out well) for the whole lattice community and hope we will be able to count on the support of all lattice collaborations.

\section{Acknowledgments}

I thank the organizers for the invitation and an excellent organization of the conference, and my FLAG colleagues for a pleasant collaboration and for reading the manuscript - in particular Heiri Leutwyler and Tassos Vladikas.

\footnotetext{
${ }^{2}$ The WG on semileptonic $B$ decays has four members, but only three of them belong to lattice collaborations.
} 


\section{References}

[1] LHCb Collaboration Collaboration, R. Aaij et. al., Evidence for CP violation in time-integrated $D^{0} \rightarrow h^{-} h^{+}$decay rates, Phys.Rev.Lett. 108 (2012) 111602, [arXiv:1112.0938].

[2] G. Colangelo, S. Dürr, A. Jüttner, L. Lellouch, H. Leutwyler, et. al., Review of lattice results concerning low energy particle physics, Eur.Phys.J. C71 (2011) 1695, [arXiv: 1011.4408 ].

[3] Particle Data Group Collaboration, J. Beringer et. al., Review of Particle Physics (RPP), Phys.Rev. D86 (2012) 010001.

[4] [RBC/UKQCD 10], P. A. Boyle, et. al., $K \rightarrow \pi$ form factors with reduced model dependence, Eur. Phys. J. C69 (2010) 159-167, [arXiv: 1004 .0886].

[5] [RBC/UKQCD 07], P. A. Boyle, et. al., $K_{l 3}$ semileptonic form factor from $2+1$ flavour lattice QCD, Phys. Rev. Lett. 100 (2008) 141601, [arXiv: 0710 . 5136].

[6] [ETM 10D], V. Lubicz, F. Mescia, L. Orifici, S. Simula, and C. Tarantino, Improved analysis of the scalar and vector form factors of kaon semileptonic decays with $N_{f}=2$ twisted-mass fermions, PoS LAT2010 (2010) 316, [arXiv: 1012 .3573].

[7] [ETM 09A], V. Lubicz, F. Mescia, S. Simula, and C. Tarantino, $K \rightarrow \pi \ell v$ semileptonic form factors from two-flavor lattice QCD, Phys. Rev. D80 (2009) 111502, [arXiv: 0906.4728 ].

[8] [QCDSF 07], D. Brömmel, et. al., Kaon semileptonic decay form factors from $N_{f}=2$ nonperturbatively $O($ a)-improved Wilson fermions, PoS LAT2007 (2007) 364, [arXiv: 0710 . 2100].

[9] [RBC 06], C. Dawson, T. Izubuchi, T. Kaneko, S. Sasaki, and A. Soni, Vector form factor in $K_{l 3}$ semileptonic decay with two flavors of dynamical domain-wall quarks, Phys. Rev. D74 (2006) 114502, [hep-ph/0607162].

[10] [JLQCD 05], N. Tsutsui, et. al., Kaon semileptonic decay form factors in two-flavor QCD, PoS LAT2005 (2006) 357, [hep-lat/ 0510068$].$

[11] [ETM 10E], F. Farchioni, G. Herdoiza, K. Jansen, M. Petschlies, C. Urbach, et. al., Pseudoscalar decay constants from $N_{f}=2+1+1$ twisted mass lattice QCD, PoS LAT2010 (2010) 128, [arXiv:1012.0200].

[12] [MILC 10], A. Bazavov, et. al., Results for light pseudoscalar mesons, PoS LAT2010 (2010) 074, [arXiv:1012.0868].

[13] RBC Collaboration, UKQCD Collaboration Collaboration, [RBC/UKQCD 10A], Y. Aoki, et. al., Continuum Limit Physics from 2+1 Flavor Domain Wall QCD, Phys.Rev. D83 (2011) 074508, [arXiv:1011.0892].

[14] [BMW 10], S. Dürr, et. al., The ratio $F_{K} / F_{\pi}$ in QCD, Phys. Rev. D81 (2010) 054507, [arXiv:1001.4692].

[15] [JLQCD/TWQCD 09A], J. Noaki, et. al., Chiral properties of light mesons with $N_{f}=2+1$ overlap fermions, PoS LAT2009 (2009) 096, [arXiv : 0910 . 5532].

[16] [MILC 09A], A. Bazavov, et. al., MILC results for light pseudoscalars, PoS CD09 (2009) 007, [arXiv:0910.2966].

[17] [MILC 09], A. Bazavov, et. al., Full nonperturbative QCD simulations with 2+1 flavors of improved staggered quarks, Rev. Mod. Phys. 82 (2010) 1349-1417, [arXiv: 0903.3598$].$ 
[18] [Aubin 08], C. Aubin, J. Laiho, and R. S. Van de Water, Light pseudoscalar meson masses and decay constants from mixed action lattice QCD, PoS LAT2008 (2008) 105, [arXiv : 0810 . 4 328].

[19] [PACS-CS 08], S. Aoki, et. al., 2+1 Flavor lattice QCD toward the physical point, Phys. Rev. D79 (2009) 034503, [arXiv:0807.1661].

[20] [PACS-CS 08A] and Y. Kuramashi, PACS-CS results for $2+1$ flavor lattice QCD simulation on and off the physical point, PoS LAT2008 (2008) 018, [arXiv: 0811 . 2630].

[21] [RBC/UKQCD 08], C. Allton, et. al., Physical results from 2+1 flavor domain wall QCD and SU(2) chiral perturbation theory, Phys. Rev. D78 (2008) 114509, [arXiv: 0804 . 0473 ].

[22] [HPQCD/UKQCD 07], E. Follana, C. T. H. Davies, G. P. Lepage, and J. Shigemitsu, High precision determination of the $\pi, K, D$ and $D_{s}$ decay constants from lattice QCD, Phys. Rev. Lett. 100 (2008) 062002, [arXiv:0706.1726].

[23] [NPLQCD 06], S. R. Beane, P. F. Bedaque, K. Orginos, and M. J. Savage, $f_{K} / f_{\pi}$ in full QCD with domain wall valence quarks, Phys. Rev. D75 (2007) 094501, [hep-lat / 0606023 ].

[24] [MILC 04], C. Aubin, et. al., Light pseudoscalar decay constants, quark masses, and low energy constants from three-flavor lattice QCD, Phys. Rev. D70 (2004) 114501, [hep-lat/ 0407028 ].

[25] [ETM 09], B. Blossier, et. al., Pseudoscalar decay constants of kaon and D-mesons from $N_{f}=2$ twisted mass Lattice QCD, JHEP 07 (2009) 043, [arXiv : 0904.0954 ].

[26] [QCDSF/UKQCD 07], G. Schierholz et al., Probing the chiral limit with clover fermions I: The meson sector, talk given at Lattice 2007, Regensburg, Germany, PoS LAT2007, 133.

[27] M. Antonelli et. al., An evaluation of $\left|V_{u s}\right|$ and precise tests of the Standard Model from world data on leptonic and semileptonic kaon decays, Eur. Phys. J. C69 (2010) 399-424, [arXiv: 1005 . 2323 ].

[28] [LR 84], H. Leutwyler, and M. Roos, Determination of the elements $V_{u s}$ and $V_{u d}$ of the Kobayashi-Maskawa matrix, Z. Phys. C25 (1984) 91.

[29] P. Post and K. Schilcher, $K_{l 3}$ form factors at order $p^{6}$ in chiral perturbation theory, Eur. Phys. J. C25 (2002) 427-443, [hep-ph/0112352].

[30] J. Bijnens and P. Talavera, $K_{l 3}$ decays in chiral perturbation theory, Nucl. Phys. B669 (2003) 341-362, [hep-ph/0303103].

[31] M. Jamin, J. A. Oller, and A. Pich, Order $p^{6}$ chiral couplings from the scalar K $\pi$ form factor, JHEP 02 (2004) 047, [hep-ph/ 0401080$].$

[32] V. Cirigliano et. al., The Green function and SU(3) breaking in $K_{l 3}$ decays, JHEP 04 (2005) 006, [hep-ph/0503108].

[33] A. Kastner and H. Neufeld, The $K_{l 3}$ scalar form factors in the Standard Model, Eur. Phys. J. C57 (2008) 541-556, [arXiv:0805.2222].

[34] J. C. Hardy and I. S. Towner, Superallowed $0^{+} \rightarrow 0^{+}$nuclear $\beta$ decays: A new survey with precision tests of the conserved vector current hypothesis and the Standard Model, Phys. Rev. C79 (2009) 055502, [arXiv:0812.1202].

[35] E. Gamiz, M. Jamin, A. Pich, J. Prades, and F. Schwab, Theoretical progress on the $V_{\text {us }}$ determination from $\tau$ decays, PoS KAON (2008) 008, [arXiv: 0709.0282$].$

[36] K. Maltman, C. E. Wolfe, S. Banerjee, I. M. Nugent, and J. M. Roney, Status of the hadronic $\tau$ decay determination of $\left|V_{u s}\right|$, Nucl. Phys. Proc. Suppl. 189 (2009) 175-180, [arXiv: 0906.1386 ].

[37] J. Laiho, E. Lunghi, and R. S. Van de Water, Lattice QCD inputs to the CKM unitarity triangle analysis, Phys. Rev. D81 (2010) 034503, [arXiv: 0910 .2928]. 